\section{Cycad Toxins and Neurological Diseases in Guam: Defining Theoretical and Experimental Standards for Correlating Human Disease with Environmental Toxins}

\author{
Thomas E. Marler ${ }^{1}$ \\ College of Natural and Applied Sciences, University of Guam, UOG Station, \\ Mangilao, Guam 96923
}

Vivian Lee and Christopher A. Shaw

Department of Ophthalmology, University of British Columbia, Vancouver, $B C$, Canada

Additional index words. ALS-PDC, BMAA, cycad, Cycas micronesica, Guam, neurotoxins, secondary compounds, steryl glucosides

\begin{abstract}
Consumption of Cycas micronesica seed tissue has been associated with the amyotrophic lateral sclerosis-parkinsonism dementia complex (ALS-PDC) of the Western Pacific. However, failures to document vital plant and neighborhood descriptors and pronounced variability in toxin concentrations noted within and among studies obfuscate decades of research on this subject. We discuss the theoretical and experimental constraints of plant tissue sampling in relation to human disease research. Comparisons are made between this approach and methods used throughout the history of ALS-PDC research, most notably very recent reports concerning $\beta$-methyl-amino-alanine. Methods for studying possible plant neurotoxins need to be standardized and must follow rigorous criteria to be valid in principle. Our discussions reveal why these criteria are essential and highlight the impact that natural variations have on environmental toxin quantification and interpretation. Past research on cycad toxins is deficient on experimental and theoretical grounds, and interpretation of published data is dominated by ambiguities. This area of study as conventionally conceived and carried out needs transforming. We argue that future empirical studies should honor appropriate plant science standards concomitantly with medical science standards. This dual focus will ensure appropriate sampling scheme, sample size, and reporting of background plant and community factors known to influence phenotypic plasticity.
\end{abstract}

The island of Guam claims an endemic cycad species and an unwelcome high historical incidence of neurological disease among the Chamorro residents. The first written record suggesting that consumption of the cycad seed tissue was a probable cause of the neurological diseases was made by Spanish Lieutenant Colonel Felipe de la Corte in 1865 (as cited by Rogers, 1995). His opinions were ignored until the mid-20th century when the disease now known as ALS-PDC and the cycad now known as Cycas micronesica K.D. Hill first received joint attention from the medical research community (reviewed in Kurland, 1988; Kurland et al., 1994; Mabry, 2001). Consumption of cycad megagametophyte tissue as processed flour was commonly practiced, and the resulting inadvertent ingestion of cycad toxins was considered the vehicle for human exposure (Whiting, 1963). Numerous articles and review chapters on cycad

Received for publication 29 Dec. 2004. Accepted for publication 27 Apr. 2005. We thank J. Chung, C. Melder and N. Dongol for assistance. Support provided by USDA CSREES Special Grant in Tropical/ Subtropical Agricultural Research to TEM and U.S. Army Medical and Materiel Command (DAMD1702-1-0678), NSERC Canada, and Scottish Rite Charitable Foundation of Canada to CAS

${ }^{1}$ Corresponding author; e-mail tmarler@uog.edu. toxins have been published throughout recent decades, and many of these were in pursuit of a reasonable link between exposure to cycad compounds and the prevalence of ALS-PDC (e.g., Kurland, 1993 and citations therein). However, no article within this sizeable body of literature adequately described field sampling methods, habitat, conspecific and interspecific neighbors of sampled plants, scaling and allometric relations, plant lifehistory characteristics and events, and other factors that are known to influence secondary metabolism of plants. Unfortunately, this assertion holds true for the most recent publications in this field (e.g., Banack and Cox, 2003; Yagi, 2004), despite the exposure of this manifest shortcoming of inadequate sampling methods by Zhang et al. (1996).

The endemic C. micronesica has not been popular in Guam's horticulture industry. Alternatively, $C$. revoluta Thunb. is one of the most common landscape specimens in the commercial and residential landscape. Our recent surveys revealed that the purported link between the native cycad and the dreaded human disease is partly to blame for the lack of interest in using this handsome plant in the local horticulture industry. As a result, unanswered questions surrounding the hypothesis that cycad toxin exposure is causal for ALS-PDC must be put to rest before the horticulture industry can expect increased use of this magnificent specimen plant.

One troublesome facet of past research has been the highly variable concentration of various toxins from batch to batch within a study, and from study to study within and among research teams (e.g., Palm and Cycad Societies of Australia, 1993, and citations therein). Indeed, this variability has been a reason for failures to embrace the cycad hypothesis. However, this variability need not be viewed through the eyes of a skeptic using an enigmatic filter. We believe the documented variability would have been explicable if background biotic and abiotic habitat factors had been recorded, and appropriate sampling procedures had been used.

We focus on several steryl glucoside subtypes in our research (see Marler et al., 2005) and the present paper for two reasons. First, other known cycad toxins such as methyl azoxylmethanol(MAM) and $\beta$-methyl-amino-alanine (BMAA) have been studied for decades in spite of the fact that most do not generate behavioral or pathological outcomes that resemble ALS-PDC (reviewed by Wilson et al., 2002). Our current work strongly implicates steryl glucoside variants as contributing to ALS-PDClike outcomes in cycad fed mice (Khabazian et al., 2002; Shaw and Wilson, 2003; Wilson et al., 2002), mirroring in vivo data obtained by earlier researchers (Ambike and Rao, 1967). Second, the well-known cycad toxins such as MAM and BMAA are removed from cycad tissue during the water-leaching used in traditional flour preparation methods, but the steryl glucosides are insoluble in water. Our ongoing researchincludes all known neurotoxins in cycad tissue, since the role of these metabolites in cycad plants has never been addressed in past research (Brenner et al., 2003). Theoretical and experimental standards described in the present study will be used to define procedural and conceptual concerns as other candidate toxins are given full scrutiny.

Our aim in the present paper is to describe the multitude of omissions and shortcomings that have defined historical and ongoing research methods in this field and illustrate the need for appropriate theoretical and experimental directions in future research to remove the ambiguities that are defining of this large literature base. Modifying the patterns of past research addressing the link between the Guam cycad and Guam's neurological diseases to include appropriate plant science standards is critical for gaining a complete understanding of the chemical ecology in these stunning plants. We begin with two sections discussing the various plant and habitat characteristics that likely influence phenotypic plasticity in cycad biochemistry, factors that have been ignored in past research.

Ignoring what Cannot be Ignored: Aerial Factors

Control over known and probable biases must be included in the sampling protocols in environmental research to ensure accuracy and 
repeatability. Following is a short list of factors that define the aerial environment of cycad plants and that elicit phenotypic plasticity of plant secondary chemistry. These are the major factors that we have documented and standardized for our own research (Marler et al., 2005). We suggest documenting each of these factors as a minimum for reliable interpretation of future research on cycad secondary metabolism.

The chemical constituency of a plant organ observed at one point in time is the result of many processes in plant life history. For concentration, pool size, and allocation of secondary compounds among organs of cycad plants, history of herbivory may elicit immense influence on instantaneous metabolite concentration. For example, herbivory may increase the products of secondary metabolism of some species so strongly that fitness is compromised (Heil and Baldwin, 2002). Moreover, herbivory of leaf tissue may elicit a plastic response of secondary compounds found in reproductive structures (Baldwin and Karb, 1995). No testing has been conducted to determine the direction and magnitude of plastic responses of cycad tissue to herbivory. Until this testing is completed, a detailed description of herbivory history as a minimum measure of precaution is essential to account for consumer damage biases. This cannot occur without a lengthy history of observing the plants that are sampled.

A second factor in plant life history that must be known to control background bias when quantifying secondary compounds in cycad plants is age of reproductive flushes that are present on sampling dates. In Guam's environment, the exterior of a C. micronesica seed changes from light green initially through a dark green phase to bronze by ca. 8 months of age (T. Marler, unpublished data). Sun exposure influences the rate of this color change. Many seeds do not increase in volume beyond 8 months, and exterior phenotype changes little until the sarcotesta becomes dark brown before seed abscission. The timing of this change from bronze to brown is highly variable among plants, but occurs from 17 to 21 months under typical conditions (T. Marler, unpublished). Thus, the exterior phenotype of seeds may appear similar from 8 months to greater than 21 months. Our ability to estimate age of seeds observed on previously unvisited plants is nil within this age range, despite our extensive experiences in observing behavior of the species since 1997 . Our ability to estimate age of seeds after the color transition from bronze to dark brown is also nil, despite the fact that this transition occurs as early as 17 months yet seeds may remain on plants $>30$ months.

Our research to date(Marleretal., 2005) does not allow a definitive assessment of seed age and secondary metabolism, since initial objectives were designed to define methods for sampling schemes. However, a comparison of the means from the 12-month-old tissue with the means from 17- or 19-month-old megagametophyte tissue indicate the younger seeds contain greater concentration of steryl glucosides (see Marler et al., 2005). These observations are evidence that seed age must be known for repeatability and removal of ambiguity, but also that further research to clarify the influence of seed age on neurotoxin concentration is warranted. Seed age has never been reported in past publications in this area of research.

The timing and magnitude of resource allocations within a plant are determined by modular organization, with each growth module behaving semi-autonomously (Herms and Mattson, 1992). The synchrony of these growth modules among C. micronesica individuals within a landscape is increased by typhoon damage (Hirsh and Marler, 2002). With each successive growth module thereafter, the population becomes less synchronized. The initial cost of constructing a leaf is generally greater for species with evergreen leaves, large leaves, and leaves that are sclerophyllous (Chabot and Hicks, 1982). Cycas micronesica leaves are distinguished by all three of these characteristics. Although no resource budgets have been determined to date to quantify these dynamics for cycads, the substantial costs and payback time no doubt influence the partitioning of assimilates. We suggest a third factor of plant life history that must be recorded to obtain unambiguous data is the growth history of these episodic events before sampling dates. Timing of past growth flushes has never been reported in past publications in this area of research.

Scaling relations among vegetative organs at the individual plant level follow consistent patterns that apply across species and ecological conditions (Niklas, 2004). Likewise, reproductive growth size may scale with plant size for a broad taxonomic range that includes angiosperm and nonangiosperm species (Niklas, 1993; Peters et al., 1988). Zotz (2000) reported that lack of attention to plant size in physiology studies has the potential to introduce inappropriate ambiguities in interpretation of results. The relationship between reproductive growth characteristics and plant size is unknown for cycad plants. Until more is learned about distributional biases of secondary compounds among competing organs and the influence of plant size on theses biases, we suggest that height and any measure of stem girth are minimum requirements to account for these mass- or volume-related sample biases. No measure of plant size has been included in past publications in this area of research.

Crop load has been studied extensively in fruit science, and many horticultural practices in orchard management are based on this knowledge (e.g., Childers, 1995). The crop load affects varied characteristics such as distribution of assimilates, fruit size, fruit chemistry, and total plant yield. Position of fruits within an inflorescence or within a canopy affects maturation and sampling procedures across a wide range of architecture types (De Silva et al., 2000; Miles et al., 1996; Viti et al., 2003). Despite this broad knowledge base indicating fecundity and location of a reproductive organ within the plant influence many characteristics, past ALS-PDC researchers have ignored the need to document this information from sampled cycad plants. We suggest that recording the number of seeds on a sampled plant should be treated as a minimum measure of precaution to account for probable seed load biases.

The level of sun exposure at the whole plant level is controlling of many plastic responses, including synthesis and allocation of secondary compounds (e.g., Callaway et al., 2003; Herms and Mattson, 1992). Moreover, the level of incident light at microsites within a plant canopy is known to influence reproductive growth characteristics of those microsites (Childers, 1995). For example, seeds on the north and south orientations of a cycad plant may experience less incident light than on the east and west orientations. Moreover, influence of shade from neighboring plants on cycad secondary metabolism is unknown. We suggest that recording incident light or percent shade is required to establish legitimacy of a data set. Historical ALS-PDC research has ignored this requirement.

Comparing a compound in tissue from one plant with that in tissue from another plant without ensuring the shade level of each plant is homogeneous (e.g., Banack and Cox, 2003) cannot be justified. In fact, one of the underlying justifications Bryant et al. (1983) used to propose the carbon/nutrient balance hypothesis was the known influence of shade on phenotypic variation in secondary metabolism.

Addressing the above-ground biases. Throughout the decades of research on C. micronesica secondary metabolites, $100 \%$ of the articles omitted essential information about plant life history, size, fecundity, and sun exposure. Considering the fact that phenotypic plasticity of plants in response to these and other factors may cause characteristics to vary by orders of magnitude (see Callaway et al., 2003), exclusion of this information in past research is not acceptable. We propose the failure to report these influential factors may fully explain the variability in neurotoxin concentration from study to study. Certainly, future attempts to determine distribution of secondary compounds among various tissues of cycad plants must include this information for conclusions to be meaningful. More importantly, the sample size needed to ensure accuracy will be minimized by these efforts to control background biases, and this will directly improve conservation measures as this research continues.

Our approach was to use these descriptors to standardize the plants included in each experiment (see Marler et al., 2005), thereby ensuring these factors were relatively homogeneous throughout the population. An appropriate alternative strategy would be to treat the numerical data from these factors as co-factors in covariance analysis. Both approaches would remove the bias from each mean, but would not inappropriately suggest causal relationships. Our ongoing research is designed to determine the direct influence of these varied plant factors on neurotoxin concentration in C. micronesica tissue. We believe this information will lead toward an understanding of the role of these metabolites in the plant.

\section{Ignoring what Cannot be Ignored: Below-ground Factors}

ALS-PDC incidence in Guam peaked in the mid-20th century, and incidence has been declining since this time (see Kurland, 1993). 
Ongoing research designed to determine the relationship of a historical peak in cycad tissue ingestion by Guam residents several decades ago to a subsequent peak in neurological disease incidence among those residents must employ methods that are relevant to the seed collection environment during that era in history. Following is a short list of environmental factors that impact rhizosphere dynamics and are known to influence secondary chemistry of plants. We believe these factors render the use of source plants outside of native habitats of little to no value in this line of research.

Physical and chemical properties of litterfall influence myriad habitat processes that affect phenotypic plasticity of plant biochemistry (e.g., Loranger et al., 2002; Madritch and Hunter, 2002; Santa Regina and Tarazona, 2001). For example, Allison and Vitousek (2004) report a 50 -fold variation in leaflitter decay rates among 11 species, with native species exhibiting much slower decay rates than alien invader species. Our initial studies (Marler et al., 2005) are the first to ensure that interspecific neighbors of sampled plants were endemic or indigenous, and to document these providers of detritus inputs during the years before sampling dates. The relevance of past reports including compounds from tissue sampled from C. micronesica plants in ex situ botanic gardens is suspect, but even past studies that failed to document the habitat biodiversity of sampled cycad plants in Guam have ignored this relevant factor.

Detritovores and other edaphic microorganisms involved in biodegradation of detritus and biosynthesis of glomalin and other chemicals that enter the soil matrix are critical in defining soil physicochemical properties and cycling of abiotic and biotic factors in the plant's rooting environment (e.g., Caesar-TonThat and Cochran, 2001; Rillig et al., 2001). The ecological efficiency of Guam's indigenous edaphic microorganisms in relation to those that happen to surround the rooting environment of a cycad plant via anthropogenic actions in $e x$ situ botanic gardens is virtually unknown.

The cycad-cyanobiont relationship has unique features that include a high degree of specificity within a coralloid root structure (Costa et al., 1999) and a possible direct influence of phenolic compounds on the formation of symbiosis and metabolism (Lobakova et al., 2004). The access to fixed nitrogen afforded by this symbiosis may be one of the reasons that cycads were selected for survival throughout tens of millions of years (Norstog and Nichols, 1997). Based on the results of Ow et al. (1999), numerous Nostoc strains found in ex situ gardens would likely invade the conspicuous invasion zones of $C$. micronesica coralloid roots. However, presumption that behavior of these 'alien' cyanobionts is representative of behavior within the indigenous cycad-Nostoc association in Guam is not justified. Genetic diversity among Nostoc strains isolated from cycad species within their natural range is not known to date, and caution should prevail until this diversity is known. Indeed, Nilsson et al. (2000) reported that genotype of cyanobiont isolates from Gunnera species grouped by geographic region, indicating a limited distribution of the closely related strains that formed each geographic group.

A Nostoc strain isolated from symbiotic tissue and maintained in axenic cultures cannot be considered a germane strain without subsequent validation. Isolation of an inconsequential symbiont or nonsymbiotic Nostoc from the symbiotic plant tissue is likely. For Azolla-Nostoc symbioses (e.g., Gebhardt and Nierzwicki-Bauer, 1991), isolated Nostoc strains were regarded and studied as relevant Azolla symbionts until proper examination ultimately proved they were distinct from major symbionts. Isolated Nostoc strains from C. micronesica plants grown in ex situ gardens and maintained as axenic cultures (e.g., Murch et al., 2004) are not only alien genotypes, but should justifiably be treated as immaterial to the symbiotic system until they have been proven to be major symbionts.

In addition to cyanobionts, cycad roots are also proficient hosts of mycobionts (Fisher and Vovides, 2004; Muthukumar and Udaiyan, 2002). Thus, the cycad symbiotic system is actually a tripartite system, where each organism interacts with the other two. Research on physiology of this system must address the interactions among all three organisms (e.g., Tsimilli-Michael et al., 2000). The many issues discussed above concerning relevance of 'alien' detritus providers, soil microorganisms, and cyanobionts also applies to mycorrhizae that colonize C. micronesica roots in an ex situ garden. In light of the tripartite system, however, the lack of relevance of ex situ research may be even more magnified. Indeed, both of the microbionts in ex situ sites are distinct from those that comprise the indigenous tripartite system in Guam's environment. Knowledge of the cycad-mycorrhizae tripartite system with Nostoc is lagging behind that of the legume-mycorrhizae tripartite system with nodule-inducing bacteria, but this does not justify ignoring the likelihood that the three-way interactions are as controlling of cycad growth and development as are those in legumes. Fisher and Vovides (2004) point out the probability that cycads may require mycorrhizal colonization for realistic Nostoc function.

We use these truths to suggest biochemistry of C. micronesica in the absence of indigenous microbionts must be viewed as artifacts until genetic and phenotypic geographic distributions of the microbiont populations are determined. To illustrate, indigenous cyanobionts and mycobionts could be isolated from a Guam habitat and transported to a remote garden setting. These microbionts could then be used to inoculate specimens of C. revoluta or any other Cycas species to develop a functioning tripartite system. Reasonable discussion of biochemistry data from this tripartite system would not infer that the results relate to biochemistry of an indigenous C. micronesica tripartite system in habitat, even though two of the three organisms would be indigenous to Guam. Similarly, reasonable discussion of biochemistry data from an ex situ C. micronesica specimen in a tripartite system with two alien microbionts would not infer that the results are relevant to the real world.
The complexities of how conspecific and interspecific neighbors influence a plant's growth and physiology via below-ground communication are beginning to be understood (reviewed in Callaway, 2002; Callaway et al., 2003). Recent evidence reveals sophisticated plant root communication dexterity that includes an ability to distinguish conspecific roots from self roots (e.g., Falik et al., 2003; Gersani et al., 2001). These root interactions do not elicit straightforward plant responses resulting from a neighbor's competitive or facilitation attributes. Rather, a suite of beneficial and negative effects of a plant's habitat interplay spatially and ephemerally to determine the net-positive or net-negative effect of conspecific and interspecific neighbors (Holzapfel and Mahall, 1999). Positive attributes of close neighbors are being tapped to modify traditional reforestation efforts through use of facilitating nurse plants (e.g., Castro et al., 2002).

Secondary metabolism is included in the assemblage of responses under the influence of neighbors. For example, allocation costs to secondary compounds of Nicotiana attenuata were not observed in the absence of neighbors, but were extensive when plants were grown with neighbors in environmentally realistic conditions (van Dam and Baldwin, 2001). Ehlers and Thompson (2004) report that co-occurring plant species adapt to one another. No doubt the multifaceted levels of root communication combine with the chemical relations of detritus inputs by neighbors to select for this evolutionary phenomenon. These root interactions among co-occurring plants indicate the biochemistry of $C$. micronesica plants growing in remote locations devoid of indigenous neighbors cannot be considered relevant to biochemistry of these plants in habitat where Guam's residents harvested seeds for human consumption several decades ago.

Edaphic factors within the natural range of a cycad species provided many of the evolutionary selective pressures that define phenotype and genotype. Moreover, minimal differences in habitat abiotic factors may profoundly modify the complex positive and negative interactions among plant neighbors (Choler et al., 2001; Schenk et al., 2003). Thus, transfer of the entire suite of indigenous organisms inclusive of those listed above to a remote garden would still fall short of providing the basics of an experimental setting from which relevant physiology data could be obtained. Transfer of the edaphic substrates that serve as the matrix for the fine-tuned biological interactions known to influence plant physiology would also be required.

The Guam habitats that $C$. micronesica has colonized are varied. The major island perimeter forested habitats are from Ritidian soil series, the interior habitats in northern Guam are from Guam soil series, the upland habitats in southern Guam are from Akina soil series, and the ravine forests in southern Guam are dominated by Agfayan soil series (Young, 1988). These substrates and their rock outcrops are highly contrasting in chemistry and physical characteristics. The Ritidian and Guam soil series are both alkaline and contigu- 
ous in some areas. The slightly acid Agfayan series is contiguous with the highly acid Akina series in many locations. Thus, gene flow may be substantial between the habitats that cover a gradient overlapping the contiguous geologic substrates. Indeed, transects in two of the five habitats we have been studying since 2002 include these gradients. However, the habitats on Guam soil series are disjunct from habitats on Agfayan or Akina series, and habitats on Ritidian series are disjunct from habitats on Agfayan series. Not only are the physicochemical characteristics highly contrasting, the geographical isolation of the cycad habitats based on the contrasting substrates adds to the possibility that diversity of fragmented cycad populations is clustered genetically. Numerous plants have evolved specific genotypes that are adapted to their microenvironment more so than conspecific individuals from other microenvironments (Marler, 2002). Moreover, there is no reason to assume the microbionts from C. micronesica originating from Guam's alkaline soils are symbiotically active in Guam's acid soils, or vice versa. Similarly, there is no reason to assume the influences of interspecific neighbors found in alkaline soil habitats are similar to those influences in acid soil habitats, and vice versa.

These realities shape appropriate C. micronesica research in two manners. First, research on Guam must document habitat descriptors for methods to be repeatable and interpretation of biochemistry data to be meaningful. For example, Brenes-Arguedas and Coley (2005) report that many environmental factors explained spatial variation of secondary chemistry of Quararibea asterolepis Pitt. at the landscape level. Second, the use of individuals in ex situ collections is not advisable based on genetic fundamentals, unless microsite and soil type of the original seed collection in Guam is described in detail for each accession. Many of the provinces, especially in southern Guam, contain more than one of these soil series, and a record of the province of seed origin is insufficient.

Plants growing in sites with Akina soils must contend with a rhizosphere that is characterized by overabundance of various metals and aluminum toxicity tendencies. Indeed, these chemical characteristics of Akina soils may be one of the major reasons that some indigenous species do not occur in habitats on this substrate (Marler and Lawrence, 2004). The possibility that aluminum, iron, or other soil-derived metal ions interact with primary toxins in diet-induced pathology (e.g., Garruto et al., 1989; Zhang et al., 1996) cannot be discounted. Thus, the overabundance of these metals in the Akina soil series in relation to the other soils series in Guam may add another variable that complicates interpretation of past publications. This is yet another example of why microsite descriptions are needed to authenticate each study.

Guam's seasonal changes are under the direct influence of heterogeneous rainfall patterns. August through October generally receive $>30 \mathrm{~cm}$ of precipitation per month, and February through April receive $<10 \mathrm{~cm}$ per month
(Young, 1988). These seasonal differences in water availability exert considerable control over plant growth and development, and soil characteristics largely define the severity of seasonal drought stress. Habitats in Ritidian and Guam series soils dry rapidly following rainfall, but habitats in Akina and Agfayan series soils retain water reserves. Until more is known about cycad secondary metabolism and environmental cues such as seasonal water availability and diel or seasonal temperature patterns, research should be confined to Guam's environment and dates of sampling must be recorded. With this information documented, historic climatological data can be retrieved to draw conclusions about the influence of weather patterns before sampling dates from previous reports.

Addressing the neighborhood biases. A review of the collective literature on cycad toxins reveals that $100 \%$ of these articles fail to account for these environmental factors that largely influence plant physiology. These omissions have the potential to render these former studies irrelevant to the questions being studied or incomplete at best. Plant secondary chemistry is determined not only by intrinsic genetic and phenotypic components, but also by many community-level components. The environmental factors described above and many others clearly reveal a plant's chemical ecology is played out in a world much larger than the plant itself. For example, Hevia et al. (1999) argue that nutrient use efficiency can be validly studied only when comparing species that co-occur in the same habitats. In this context, Norstog and Nicholls (1997) warned that studying growth characteristics of cycad specimens in ex situ cultivation is of little value for making inferences about the behavior of a species in its natural habitat. They extend this warning to include a cycad specimen growing in a site within its natural range that may have had anthropogenic influences in past or present.

These warnings ring true for Guam because C. revoluta plants have been introduced to the island for many decades (Stone, 1970). This cycad species is currently a ubiquitous member of the urban landscape. The vast majority of these specimens were brought to Guam as landscape-ready container specimens from offisland nurseries. In all likelihood, alien Nostoc and mycorrhizae strains accompanied these alien cycads during the high volume imports geared toward satisfying the landscape industry. Thus, any C. micronesica in the urban landscape may be colonized by these alien strains of microbionts, and in all likelihood would be supplying experimental artifacts if they were targeted as the source of seeds for studying the relationship between cycad toxins and ALS-PDC.

Certainly ex situ collections are invaluable for protecting and preserving genetic diversity, for display purposes, and for research that is not strongly influenced by genetic $\times$ environment interactions. But plant physiology is under utmost control of environment and genetic $\times$ environment factors. Thus, physiology research in off-site collections must be restricted to appropriate objectives. For example, Marler and Willis (1997) compared leaf physiology of representatives from 10 cycad genera under homogeneous edaphic and climatic conditions. Indeed, addressing an objective this restrictive requires an ex situ collection, since no cycad habitat worldwide includes these genera in close proximity. But this research must carry the caveat that no relevance to plant behavior in habitat is inferred.

An overview of the cycad environment in the ex situ settings sampled by Banack and Cox (2003) provide a working example. The two Florida botanic gardens from which samples were obtained use many preplant procedures that create a rooting environment more heavily influenced by anthropogenic actions than geologic actions. Individual plants are grouped with conspecific neighbors in monoculture planting beds, or are surrounded by alien species including judiciously managed turfgrass. The planting sites are less shaded than Guam's cycad habitats. All plants receive routine fertilizer applications. The use of tissue from plants that have received routine doses of fertilizers to study secondary metabolism cannot be reasonably viewed as appropriate, unless the research questions are restricted to the influences of differential fertilization. One of the underlying justifications Bryant et al. (1983) used to propose the carbon/nutrient balance hypothesis was the well-known influence of fertilizers on phenotypic variation in secondary metabolism. Lastly, these Florida sites are ground zero for the American invasion of Aulacaspis yasumatsui Takagi, the Asian cycad scale that was first discovered there before 1996 (Howard et al., 1999). All of the Cycas plants in one of the ex situ sites receive comprehensive sprays of a growth regulator as a portion of the methods used for control of this deadly scale (J. Haynes, personal communication). Virtually nothing is known about the impact of synthetic pesticide spray materials on secondary metabolism of C. micronesica.

The preceding paragraph provides graphic evidence that biochemistry of tissue collected from plants in botanic gardens cannot be considered relevant to a plant's native environment. Ignoring this reality is an atrocity when tissues from some plants that have received extensive horticultural inputs are compared with tissues from other plants that have not (e.g., Banack and Cox, 2003). This approach is not consistent with scientific standards, and variation among sample means is likely more influenced by the heterogeneous anthropogenic treatments among the sample sites than inherent variation among organ and tissue categories.

One other item that requires attention is sexual compatibility. Guam's cycad is a member of the $C$. rumphii species subsection, a complex uniquely defined by the presence of a spongy flotation layer within the seed [Hill, 1994; Marler et al., 2005 (Fig. 1B)]. Little is known about compatibility among the various species that comprise this subsection, but cycads have weak fertility barriers (Hill, 1993) and compatibility among some or all of these species is highly likely (K. Hill, personal communication). For this reason we are discouraging the introduction of any of these species to the 
Guam landscape industry (Marler, 2002). The 'civilian' gene pool in Guam is unadulterated, but introducing a sexually compatible species carries the risk of escaped alien genes taking up residence in the $C$. micronesica population in habitat. Specimens of C. micronesica plants in ex situ collections are surrounded by other Cycas species that may be sexually compatible. Wind pollination has not been disproven for Cycas species, and the likelihood of genetic contamination cannot be ignored. The chance of harvesting interspecific Cycas seed material in ex situ settings does not allow their use in studies of secondary compounds in a manner that infers relevance to in situ locales.

Clearly, studying cycad specimens in native locales is the sole valid approach for determining secondary metabolism in an unambiguous manner. The use of in situ plants for this research also mandates two further considerations. First, appropriate in situ methods must exclude cycad individuals positioned within the sphere of influence of any alien arthropod or plant species. These alien organisms were not likely influencing cycad plant chemistry several decades ago when the human ingestion of seed flour reached a peak. This is becoming increasingly difficult as more alien species become established in the Guam environment, and points to the urgency of continuing this research in a timely manner. Second, since the geologic substrates and interspecific indigenous neighbors that define Guam's cycad habitats are highly contrasting, these habitat descriptors must accompany each sampling to ensure reliability. Throughout the long history of ALS-PDC research until the present study, $100 \%$ of the articles provided no information on this subject.

\section{Past Oversights and Future Directions}

The text heretofore amply describes some pertinent plant and environmental factors that have been ignored in past research on the relationship between cycad toxins and ALS-PDC. Clearly, this line of research as conventionally conceived is in need of a major shift in methods employed. Broadly, the established pattern of zealous focus on animal toxicology and epidemiology methods while disregarding relevant plant and environmental methods needs to be reassessed. We next discuss these issues in general.

Cycas micronesica research. Cycads are the oldest living seed plants and are an opulent source of information for interpreting plant biology, yet have been largely ignored in recent developments of plant research (see Brenner et al., 2003). Similarly, Stone (1970) reported that the physiology of Guam's plants was virtually unknown, yet more than 3 decades later the number of physiology studies on Guam's endemic and indigenous plants remains in the single digits. Continued research on this valuable endemic species promises to provide much useful information about horticulture, human neurological diseases, tropical ecosystems, and chemical ecology.

The historical research on Guam's neurological diseases collectively supports several certainties at this stage. Epidemiology of these human diseases strongly suggests that exposure to an environmental factor is partly causal, $C$. micronesica tissues that have been ingested in the traditional diet contain neurotoxins that maintain integrity through the traditional flour preparation and cooking procedures, no defining plant function has been revealed for any of the studied compounds, and no compelling evidence can be used to discount the hypothesis that direct exposure to cycad compounds is the causal environmental factor (e.g., Brenner et al., 2003; Mabry, 2001). Moreover, our animal model induces neurodegeneration following exposure to cycad tissue in a ready and repeatable manner (Shaw and Wilson, 2003). If the cycad hypothesis is even marginally correct, applying results from the Guam model toward eventual prevention of many neurological diseases appears to be possible. However, ongoing research must be conducted by using appropriate plant science standards that include adequate control over sampling methods (e.g., Marler et al., 2005) and description of habitat and plant factors that have the potential to affect secondary metabolism.

Nomenclature. Recent publications (e.g., Chen et al., 2002; Cox and Sacks, 2002; Zaninovic, 2004) continue to sustain the past confusion about nomenclature of this eminent cycad. The Guam cycad is Cycas micronesica (Hill, 1994), and is a member of the C. rumphii species complex. Every member of this complex has been erroneously called C. circinalis L. or C. rumphii Miq. at some point. However, the distribution of C. rumphii is restricted to a band of island nations centered on the Moluccan island group, and the distribution of $C$. circinalis is restricted to India (Hill and Stevenson, 2005; K. Hill, personal communication, A. Lindstrom, personal communication). Cycas micronesica is endemic to Guam and surrounding islands. If considered within the broad context of various disciplines, throughout years, and among countries, the number of times the Guam cycad has been mentioned in the literature perhaps eclipses that of any other cycad species. Certainly, the continued use of incorrect names to refer to this species unduly perpetuates confusion.

The decades of extensive research on the link between cycad toxins and Guam's neurological diseases have attracted a plethora of research scientists in many fields. Until presently, this research has progressed with the exclusion of a local plant scientist. This example of continued misapplication of outdated nomenclature validates the assertions of Bawa et al. (2004) that research teams addressing topics in tropical biology need to include members of the local community.

Secondary compounds in cycads. Questions concerning the function of secondary compounds in cycads have not been addressed to date(Brenner etal., 2003). Pathogenic reactions by many herbivores have been demonstrated for many of these cycad compounds. However, that animal response is not definitive evidence that the compounds embody a defensive role or that herbivore pressures in evolutionary history elicited their selection.
Brenner et al. (2003) state that other physiological roles for BMAA should be investigated, such as possible endogenous signaling compounds. We suggest this assertion holds true for all of the identified compounds, and that the pathological responses of animals to the known C. micronesica toxins are incidental to evolutionary pressures that selected for these interesting metabolites. The continued study of secondary metabolism in cycads would be best served by answering questions within the context of the various resource models, such as the growth-differentiation balance hypothesis, the carbon-nutrient balance hypothesis, or the optimal partitioning hypothesis (Bloom et al., 1985; Herms and Mattson, 1992). These rely in diverse ways on the premise that a fundamental tradeoff in resource or ecological costs exists between growth and differentiation processes including secondary metabolism, or that response to limiting factors elicits a shift to make all resources equally limiting among tissues or processes.

The known functions of plant secondary compounds include structural support, attraction of pollinators or dispersers, temporary assimilate storage and transport, hormone regulation, and myriad other functions (e.g., Herms and Mattson, 1992). Cantatore et al. (2000) propose that steryl glucosides are carriers of glucose across membranes and serve as glucose donors. The loading of immature seed tissues with these compounds (Marler et al., 2005) lends itself to the possibility that they do indeed serve as carbohydrate donors for long-term development of tissue throughout the lengthy embryo and megagametophyte maturation period. The decline in steryl glucosides with seed age substantiates the possibility that early storage pools are metabolized to act as donors of resources. Alternatively, Kunimoto et al. (2002) provide evidence that steryl glucosides are mediators in signal transduction to induce beneficial stress responses. In general, sterols are involved in regulating membrane properties (e.g., Hartmann, 2003). All members of the C. rumphii species subsection produce seeds with a flotation layer and are designed to withstand extended periods of floating in ocean currents. The ability of these seeds to maintain viability and integrity of tissue function throughout extended exposures to extreme osmotic potentials may involve the proposed signal transduction or membrane regulating properties of these interesting compounds.

Considering the many functions of secondary compounds, we agree with Brenner et al. (2003) that searching for plant functions other than anti-herbivory functions is justified for each identified cycad metabolite. In contrast, Banack and Cox (2003) opine that the neurotoxins found in C. micronesica tissues have, indeed, evolved as anti-herbivory compounds. An abundance of BMAA in the sarcotesta is evidence they use for this opinion, since this is the tissue consumed by fruit bats. We have already argued that their data are ambiguous and statistical validity is impossible since their methods included the use of a sample size of one (Marler et al., 2005). However, our data also reveals that sarcotesta tissue is, in general, 
more heavily endowed with steryl glucosides than is megagametophyte tissue (Marler et al., 2005). We suggest that this spatial distribution does not support a chemical defensive role and offer several lines of reasoning for this suggestion.

While the cycad sarcotesta is not homologous with angiosperm pericarp, it is considered analogous in many respects (Brenner et al., 2003; Norstog and Nicholls, 1997). One of those analogies is attraction of seed dispersers. Indeed, recognizing this as an attribute of the sarcotesta is fundamental to validating the Cox and Sacks (2002) hypothesis. Preferentially loading a defensive chemical into the very tissue that attracts dispersers does not conform to a logical interpretation of a defensive strategy. On the contrary, tissues consumed by a seed disperser are equipped with a suite of biochemicals that attract and reward dispersers. For example, C. micronesica sarcotesta tissue emits pungent fruity volatiles after the seed exterior morphs from bronze to dark brown. Seeds are odorless before this phase. A sound interpretation of this characteristic is that sarcotesta from Guam's cycad is outfitted for attracting seed dispersers that are drawn by aroma rather than sight, and those volatiles are a signal to dispersers of seed maturity. Indeed, ethology of fruit bats indicates bat fruits produce large seeds with drab color and strong odors (van der Pijl, 2002). These descriptors are characteristic of $C$. micronesica seeds, and we suggest the notion of loading this tissue that attracts a disperser and is consumed by a disperser with a toxin for the purpose of deterring consumption by the disperser does not appear plausible. The presence of secondary compounds truly involved in defense are linked to increased plant fitness (e.g., Ohnmeiss and Baldwin, 2000), not linked to eliciting pathology in dispersers that improve seedling recruitment potential.

Cycad neurotoxins are unlikely candidates for defensive compounds because of failures to demonstrate direct effects on herbivore performance. The majority of research on defensive secondary chemistry has examined this direct influence on herbivore performance (Karban and Baldwin, 1997). Thus, defensive compounds are directly or indirectly linked to decreased herbivory. A foundation of the Cox and Sacks (2002) hypothesis includes long-term ingestion of sarcotesta tissue by fruit bats in order for 'biomagnification' (we believe bioaccumulation is a more appropriate term) to occur. A truly defensive chemical would deter herbivory long before bioaccumulation is given the time to transpire. Surely a compound inducing a pathological response in humans many years after ingestion is equally unlikely to demonstrate a direct effect on herbivore deterrence.

Several aspects of the BMAAbiomagnification hypothesis (Cox and Sacks, 2002) and the defensive notions of BMAA are problematic. First, the spike in incidence of ALS-PDC in the populations in Guam and Rota in the mid20 th century followed by a continuing decline in incidence of the disease indicates a decline in exposure to the causal environmental toxin since the years of highest incidence (reviewed in Kurland, 1988; Kurland et al., 1994; Mabry, 2001). This trend fits the spike in direct cycad tissue consumption during World War II years (Palm and Cycad Societies of Australia, 1993 and citations therein). Alternatively, copious consumption of Guam bats continued through the 1960s and consumption of imported bats continued through the early 1990s (Wiles, 1992). During the period $1975-89,65 \%$ of these imported bats originated in Rota, Palau, and Yap and were comprised of Pteropus mariannus, the very species of bat from Guam (Wiles, 1992). The endemic range of C. micronesica also includes all three of these islands (A. Lindstrom, personal communication). Thus, exposure to a C. micronesica toxin via bat consumption would not have declined throughout these decades of importing bats to satisfy the dietary demands of Guam residents. Neurotoxic exposure to BMAA via bat consumption clearly does not fit the patterns of disease incidence.

The original manuscript by Cox and Sacks (2002) which first proposed the hypothesis of an involvement of bats as cycad toxin vectors contained estimated historical numbers rather than real data. These authors purport to show that the decline in the bat population parallels the decline in the incidence of ALS-PDC, the latter data compiled by neuroepidemiologists working on Guam. While the human data are well established, the subjective estimations about bat population are tenuous. Scrutiny of their plots (Cox and Sacks, 2002) reveals an accounting of bat populations that span the majority of the 20th century, yet factual data points do not exist before about 1960 or subsequent to ca. 1986. The authors have drawn their graph with a sharp extrapolated upward deflection to a fabricated point of 60,000 bats in the early 1920s. They do not attempt to justify this nonexistent data point until a year later (Monson et al., 2003), when they opine that Guam could have supported this many bats based on their estimated percentage of forest coverage in the early $1900 \mathrm{~s}$. This curve forms the entire initial basis for the bat hypothesis, yet rests solely on a fabricated curve using an estimated bat population that could have been supported by an estimated forest size. Clearly without real data, there is no justifiable claim to any correlation of bat population with ALS-PDC incidence.

Scrutiny of BMAA itself reveals an extremely weak agonist at the N-methyl-D-aspartate (NMDA) receptor (Weiss et al., 1989), one of the three primary ionotropic receptors to the neurotransmitter glutamate. This classification scheme for glutamate receptor subtypes is largely independent of mammalian species. Although some differences occur in subunit composition during development, the functions, agonists, binding properties, and induced excitotoxicity are quite similar across species (Watanabe, 1996). BMAA applied to mammalian neurons in vitro evokes activation of NMDA receptors leading to an influx of $\mathrm{Ca}^{2+}$ followed by a membrane depolarization. Over-stimulation of NMDA receptors by glutamate, BMAA, or other agonists can lead to so-called excitotoxic cell death. Barring some still unknown mechanism by which a fruit bat could either sequester or detoxify BMAA, it is distinctly unlikely these mammals could accumulate high concentrations of the toxin over time without inflicting the same neural damage on themselves as has been speculated to occur in human ALS-PDC victims. Differential sequestration of BMAA in bats vs. other mammalian species is unknown and remains at best speculative. Moreover, putative detoxification mechanisms in bats would render BMAA nontoxic regardless of where the molecule next appeared in the food chain. Overall, it is difficult to conceive of a less likely candidate for neurotoxin 'biomagnification' than BMAA.

Adetailed look at partitioning of chemicals within plant tissues is useful for shedding light on putative chemical defense. For example, the steryl glucosides appear in abundance in the sclerotesta tissue (Marler et al., 2005). This tissue is analogous to the stony endocarp of a drupe, and is not at risk of herbivore consumption because of its physical characteristics. Thus, evolutionary herbivore pressures were not likely to select for preferential loading of these compounds in the sclerotesta, and their abundance in this tissue points to the probability of an alternative plant function.

Correlating human diseases with plant toxins. An emerging view in neurological disease research, particularly concerning age-dependent disorders, is that environmental factors likely play a key role in disease genesis (e.g., Shaw and Wilson, 2003). In this view, various natural and synthetic toxins, acting against a backdrop of variations in genetic susceptibility, initiate biochemical cascades which culminate in neural cell death. As discussed throughout this article, abundant evidence suggests that this is true for ALS-PDC at the very least and may be more broadly true for neurological diseases outside of Guam. A similar view concerning the impact of gene $\times$ environment interactions has been posited for various forms of cancer and cardiovascular disease (Willet, 2002).

As this nascent aspect of medical science progresses, establishing theoretical and experimental standards for correlating any environmental toxin to any human disease is warranted. We use the discussions in the present paper as an example toward this end. A wholesale focus on animal systems and disease epidemiology is not advisable if relevant botanical and habitat factors are ignored. This is precisely what has happened over the decades with the Guam cycad research. On the contrary, research addressing aspects of the environmental source of a proposed toxin should progress concomitantly with controlled research on animal responses to the toxin. For this to proceed efficiently, a plant scientist that is established in the geographic region of interest should be included in the research team (Bawa et al., 2004).

We mention one practical outcome as an example of benefits from employing this dual approach in the early stages of studying on a putative toxin. Various compounds isolated from plants may have toxic and/or therapeutic 
potentials. For example, the various steryl glucosides described in the present article appear to be toxic to neural cells, but may have quite different outcomes in other organ systems (Shaw and Wilson, 2003). The mandatory protocols using animal systems to explore the medical and therapeutic applications of plant molecules require the selective and economical harvesting of targeted molecules. This research will be ideally served by determining the most efficient and cost-effective protocols for harvesting the molecules. In our studies, the data relate to the established and ongoing research on metabolites in mature seed megagametophyte tissue, the source of flour that historically entered the human diet in Guam. However, we demonstrate that immature 1-year-old seeds may yield a greater quantity of steryl glucosides than the mature seeds that have been used throughout the decades for harvesting putative toxins (see Marler et al., 2005). We also demonstrate that sarcotesta tissue may yield a greater quantity of steryl glucosides than megagametophyte tissue. Thus, the costs of isolating and harvesting these neurotoxins for animal toxicology and epidemiology studies may be considerably reduced if protocols are modified from using mature megagametophyte tissue to including immature sarcotesta tissue.

We suggest these implications from studies extend beyond ALS-PDC research. Indeed, attempts at correlating exposure to any plant molecule with any human disease is best served by determining the most efficient and economical protocol for harvesting the molecules. This cannot be accomplished by following past examples of ignoring the importance of the plant and the plant's habitat in defining those protocols.

Zhang et al.(1996) recognized the deficiencies in past research on Guam's neurological diseases and described the need to begin documenting various aspects of cycad sample history, including specifics such as location of the sampled plant. Thus, the continued disregard for documenting and reporting these plant and habitat characteristics (e.g., Banack and Cox, 2003) is unfortunate. Our hope is our initial studies describing the need for appropriate sampling protocol (Marler et al., 2005) and the description of various factors that influence phenotypic plasticity of plants contained in the present paper validate the reality of the Zhang et al. (1996) assertions and will bring forth that needed change in the deficient methods that have plagued the ALS-PDC research since its inception.

These issues also must address the necessity of including conservation measures in all decisions. Many plant species that are of interest in horticulture, ethnobotany or medical research are threatened or endangered, and indiscriminate harvesting of tissue for isolating a metabolite cannot be justified regardless of the relative importance to human health. Certainly, research on any newly identified metabolite should address plant and habitat factors at the early stages of the research in order to fully honor mandatory conservation measures when developing strategic and efficient research protocols.
Finally, in future research designed for correlating human diseases with plant toxins, the issue of sampling error must include respect for sample size. In our initial reports (Marler et al., 2005) we obtained samples for each experiment from plants that conformed to a consistent seed age, plant size, seed load, level of canopy cover, and plant life history. Despite this focus on removing probably biases, we still observed considerable variation attributed to experimental error. We suggest the inconsistencies in past research may be entirely explained by ignoring the need to account for these sources of bias, ignoring the need for a deliberate sampling scheme, and ignoring the need to define an adequate sample size. Our results underscore the reality that secondary compounds of $C$. micronesica plants must be studied with inclusion of this information for conclusions to be accurate and consequential.

\section{Conclusions}

Methods for studying possible plant toxins need to be standardized and must follow certain rigorous criteria to be valid in principle. These criteria include testing to determine adequate sample size, honoring the need for a sampling scheme that avoids known biases, recognizing the protected and sensitive nature of cycad populations, and factoring in known plant and habitat features that influence plant secondary metabolism.

We have applied this empirical approach to our study with steryl glucoside variants (Marler et al., 2005) due to evidence that such molecules in cycad are in part causal to ALS-PDC. We suggest that this approach be viewed as mandatory for clarifying the need to establish satisfactory theoretical and experimental protocols. Our results reveal that these criteria are essential and highlight the impact that natural variations can have on toxin quantification in plants, and more importantly, on the interpretation of results.

Past research on the relationship between cycad compounds and ALS-PDC fails to meet these criteria. Throughout decades of articles on this subject, documentation of plant and habitat factors known to influence plant physiology has not occurred before our studies (Marler et al., 2005). The obvious conclusion is that researchers and reviewers have assumed these plant and environment characteristics that elicit phenotypic plasticity in plant physiology are irrelevant. Unfortunately, ongoing research continues to be plagued with these omissions. Banack and Cox (2003) present intertissue variation of several compounds by sampling individuals in different geographic locations for each tissue category, yet report no plant or landscape descriptors to allow repeatability of their methods and ensure homogeneity of these influential factors among the individuals that were sampled. Yagi (2004) presents interspecies variation of several compounds, yet reports no plant or landscape descriptors to ensure homogeneity of these influential factors among the species. Heterogeneity of plant history, fecundity, allometry, shade, ontogenesis, conspecific and interspecific neighbors, recent weather patterns, horticultural inputs, and many other factors may entirely explain their reported variation in secondary compounds. In contrast to their interpretations that tissue type (Banack and Cox, 2002) or species (Yagi, 2004) account for variation in sample group means, these undocumented plant and habitat factors that influence phenotypic plasticity may fully explain the variation in sample group means.

We argue that our approach serves as a model for defining theoretical and experimental approaches to accurately correlate any human disease with environmental toxins. The methods described will enable researchers to accurately correlate toxin distributions in the human environment with incidence of disease. For continued ALS-PDC research, other candidate neurotoxins should now be given full scrutiny using these procedural and conceptual guidelines. The failures of past ALS-PDC research teams to honor fundamental plant science standards, to adhere to acceptable sampling protocols, and to document mandatory background plant and neighborhood information cannot continue if we are to successfully use this area of study to improve human health issues.

\section{Literature Cited}

Allison, S.D. and P.M. Vitousek. 2004. Rapid nutrient cycling in leaf litter from invasive plants in Hawaii. Oecologia 141:612-619.

Ambike, S.H. and M.R.R. Rao. 1967. Studies on a phytosterolin from the bark of Ficus religiosa. Indian J. Pharmacol. 29:91-94.

Baldwin, I.T. and M.J. Karb. 1995. Plasticity in allocation of nicotine to reproductive parts in $\mathrm{Ni}$ cotiana attenuata. J. Chem. Ecol. 21:897-909.

Banack, S.A. and P.A. Cox. 2003. Distribution of the neurotoxic nonprotein amino acid BMAA in Cycas micronesica. Bot. J. Linn. Soc. 143:165-168.

Bawa, K.S., W.J. Kress, N.M. Nadkarni, and N.M. Nadkarni. 2004. Beyond paradise-Meeting the challenges in tropical biology in the 21 st century. Biotropica 36:437-446.

Bloom, A.J., F.S. Chapin, III, and H.A. Mooney. 1985. Resource limitation in plants-An economic analogy. Annu. Rev. Ecol. Syst. 16:363-392.

Brenes-Arguedas, T. and P.D. Coley. 2005. Phenotypic variation and spatial structure of secondary chemistry in a natural population of a tropical tree species. Oikos 108:410-420.

Brenner, E.D., D.W. Stevenson, and R.W. Twigg. 2003. Cycads: evolutionary innovations and the role of plant-derived neurotoxins. Trends Plant Sci. 8:446-452.

Bryant, J.P., F.S. Chapin, III, and D.R. Klein. 1983. Carbon/nutrient balance of boreal plants in relation to vertebrate herbivory. Oikos 40:357-368

Caesar-TonThat, T.C. and V.L. Cochran. 2001. Role of saprophytic basidiomycete soil fungus in aggregate stabilization, p. 575-579. In: D.E. Stott, R.H. Mohtar, and G.C. Steinhardt (eds.). Sustaining the global farm.

Callaway, R.M. 2002. The detection of neighbors by plants. Trends Ecol. Evol. 17:104-105.

Callaway, R.M., S.C. Pennings, and C.L. Richards. 2003. Phenotypic plasticity and interactions among plants. Ecology 84:1115-1128.

Cantatore, J.L., S.M. Murphy, and D.V. Lynch. 2000. Compartmentation and topology of glu- 
cosylceramide synthesis. Biochem. Soc. Trans. 28:748-750

Castro, J., R. Zamora, J.A. Hodar, and J.M. Gomez. 2002. Use of shrubs as nurse plants: A new technique for reforestation in Mediterranean mountains. Restoration Ecol. 10:297-305.

Chabot, B.F. and D.J. Hicks. 1982.The ecology ofleaf life spans. Annu. Rev. Ecol. Syst. 13:229-259.

Chen, K.-M., U.K. Craig, C.-T. Lee, and R. Haddock. 2002. Cycad neurotoxins, consumption of flying foxes, and ALS-PDC disease in Guam. Neurology 59:1664-1665.

Childers, N.F. 1995. Modern fruit science: Orchard and small fruit culture. Horticultural Publ., Gainesville, Fla.

Choler, P., R. Michalet, and R.M. Callaway. 2001. Facilitation and competition on gradients in Alpine plant communities. Ecology 82:3295-3308.

Costa, J.-L., P. Paulsrud, and P. Lindblad. 1999. Cyanobiont diversity within coralloid roots of selected cycad species. FEMS Microbiol. Ecol. 28:85-91.

Cox, P.A. and O.W. Sacks. 2002. Cycad neurotoxins, consumption of flying foxes, and ALS-PDC disease in Guam. Neurology 58:956-959.

De Silva, H.N., A.J. Hall, W.M. Cashmore, and D.W. Tustin. 2000. Variation of fruit size and growth within an apple tree and its influence on sampling methods for estimating the parameters of mid-season size distributions. Ann. Bot. 86:493-501

Ehlers, B.K. and J. Thompson. 2004. Do co-occurring species adapt to one another? The response of Bromus erectus to the presence of different Thymus bulgaris chemotypes. Oecologia 141:511-518.

Falik, O., P. Reides, M. Gersani, and A. Novoplansky. 2003. Self/non-self discrimination in roots. J. Ecol. 91:525-531.

Fisher, J.B. and A.P. Vovides. 2004. Mycorrhizae are present in cycad roots. Bot. Rev. 70:16-23.

Garruto, R.M., S.K. Shankar, R. Yanagihara, A.M Salazar, H.L. Amyx, and D.C. Gajdusek. 1989. Low-calcium, high-aluminum diet-induced pathology in cynomologus monkeys. Acta Neuropathol. 78:210-219.

Gebhardt, J.S. and S.A. Nierzwicki-Bauer. 1991. Identification of a common cyanobacterial symbiont associated with Azolla spp. through molecular and morphological characterization of free-living and symbiotic cyanobacteria. Appl. Environ. Microbiol. 57:2141-2146.

Gersani, M., J.S. Brown, E.E. O’Brien, G.M. Maina, and Z. Abramsky. 2001. Tragedy of the commons as a result of root competition. J. Ecol. 89:660-669.

Hartmann, M.-A. 2003. Sterol metabolism and functions in higher plants, p. 183-211. In: G. Daum (ed.). Topics in current genetics 6: Lipid metabolism and membrane biogenesis. SpringerVerlag, Heidelberg.

Heil, M. and I.T. Baldwin. 2002. Fitness costs of induced resistance: Emerging experimental support for a slippery concept. Trends Plant Sci. 7:61-67.

Herms, D.A and W.J. Mattson. 1992. The dilemma of plants: To grow or defend. Qrtly. Rev. Biol. 67:283-335.

Hevia, F., M.L. Minoletti O., K.L.M. Decker, and R.E.J. Boerner. 1999. Foliar nitrogen and phosphorus dynamics of three Chilean Nothofagus (Fagaceae) species in relation to leaf lifespan. Amer. J. Bot. 86:447-455.

Hill, K.D. 1993. History of the taxonomy of Australian cycads, p. 250-259. Proc. 2nd Intl. Conf. on Cycad Biology. Palm and Cycad Soc. Austral. Ltd. Milton, Queensland.

Hill, K.D. 1994. The Cycas rumphii complex (Cyca- daceae) in New Guinea and the Western Pacific. Austral. Syst. Bot. 7:543-567.

Hill, K.D and D.W. Stevenson. 2005. World list of cycads. 4 May 2005. http://plantnet.rbgsyd.gov. au/PlantNet/cycad/wlist.html.

Hirsh, H. and T. Marler. 2002. Damage and recovery of Cycas micronesica after Typhoon Paka Biotropica 34:598-602.

Holzapfel, C. and B.E. Mahall. 1999. Bidirectional facilitation and interference between shrubs and annuals in the Mojave Desert. Ecology 80:1747-1761.

Howard, F.W., A. Hamon, M. McLaughlin, T. Weissling, and S.-L. Yang. 1999. Aulacaspis yasumatsui (Hemiptera: Sternorrhyncha: Diaspididae), a scale insect pest of cycads recently introduced into Florida. Fla. Entomol 82:14-27.

Karban, R. and I.T. Baldwin. 1997. Induced responses to herbivory. Chicago Univ. Press, Chicago, Ill.

Khabazian, I., J.S. Bains, D.E. Williams, J. Cheung, J.M.B. Wilson, B.A. Pasqualotto, S.L. Pelech, R.J.Andersen, Y.-T. Wang, L. Liu, A. Nagai, S.U. Kim, U.-K. Craig, and C.A. Shaw. 2002. Isolation of various forms of steryl $\beta$-D-glucoside from the seed of Cycas circinalis: Neurotoxicity and implications for ALS-parkinsonism dementia complex. J. Neurochem. 82:516-528.

Kunimoto, S., W. Murofushi, H. Kai, Y. Ishida, A. Uchiyama, T. Kobayashi, S. Kobayashi, H. Murofushi, and K. Murakami-Murofushi. 2002. Steryl glucoside is a lipid mediator in stressresponsive signal transduction. Cell Structure Funct. 27:157-162.

Kurland, L.T. 1988. Amyotrophic lateral sclerosis and Parkinson's disease complex on Guam linked to an environmental toxin. Trends Neurosci. 11:51-53.

Kurland, L.T. 1993. Cycas circinalis as an etiologic risk factor in amyotrophic lateral sclerosis and other neurodegenerative diseases on Guam, $p$ 29-36. In: D.W. Stevenson and K.J. Norstog (eds.). Proceedings of Cycad 90, the Second International Conference on Cycad Biology. Palm and Cycad Soc. Austral., Ltd. Milton, Queensland.

Kurland, L.T, K. Radhakrishnan, D.B. Williams, and S.C. Waring. 1994. Amyotrophic lateral sclerosis-parkinsonism-dementia complex on Guam: Epidemiological perspectives, p. 109-130.In:A Williams (ed.). Motor neuron disease. Chapman and Hall, London.

Lobakova, E.S., G.A. Dubravina, and N.V. Zagoskina. 2004. Formation of phenolic compounds in apogeotropic roots of cycad plants. Russ. J. Plant Physiol. 51:486-493.

Loranger, G., J.-F. Ponge, D. Imbert, and P. Lavelle. 2002. Leaf decomposition in two semi-evergreen tropical forests: Influence of litter quality. Biol. Fertility Soils 35:247-252

Mabry, T.J. 2001. Selected topics from forty years of natural products research: Betalains to flavonoids, antiviral proteins, and neurotoxic nonprotein amino acids. J. Nat. Prod. 64:1596-1604.

Madritch, M.D. and M.D. Hunter. 2002. Phenotypic diversity influences ecosystem functioning in an oak sandhills community. Ecology 83:2084-2090.

Marler, T.E. 2002. Conservation of local genotypes when planting native plants on Guam. Micronesica Suppl. 6:55-60.

Marler, T.E and J.H. Lawrence. 2004. Chemical limitations of yoga root growth in an acid soil. Micronesica 37:157-161.

Marler, T.E., V. Lee, and C.A. Shaw. 2005. Spatial variation of steryl glucosides in Cycas micronesica plants-Within-and among-plant sampling procedures. HortScience 40(6):1607-1611.

Marler, T.E. and L.E. Willis. 1997. Leafgas-exchange characteristics of sixteen cycad species. J.Amer. Soc. Hort. Sci. 122:38-42.

Miles, D.B., G.S. Smith, and S.A. Miller. 1996 Within plant sampling procedures-Fruit variation in kiwifruit vines. Ann. Bot. 78:289-294.

Monson, C.S., S. A. Banack, and P.A. Cox. 2003 Conservation implications of Chamorro consumption of flying foxes as a possible cause of amyotrophic lateral sclerosis-Parkinsonism dementia complex in Guam. Conserv. Biology 17:678-686.

Murch, S.J., P.A. Cox, and S.A. Banack. 2004. A mechanism for slow release of biomagnified cyanobacterial neurotoxins and neurodegenerative disease in Guam. Proc. Natl. Acad. Sci. USA 101:12228-12231.

Muthukumar, T. and K. Udaiyan. 2002. Arbuscular mycorrhizas in cycads of southern India. Mycorrhiza 12:213-217.

Niklas, K.J. 1993. The allometry of plant reproductive biomass and stem diameter. Amer. J. Bot. $80: 461-467$.

Niklas, K.J. 2004. Plant allometry: is there a grand unifying theory? Biol. Rev. 79:871-889.

Nilsson, M., B. Bergman, and U. Rasmussen. 2000. Cyanobacterial diversity in geographically related and distant host plants of the genus Gunnera. Arch. Microbiol. 173:97-102.

Norstog, K.J. and T.J. Nicholls. 1997. The biology of the cycads. Cornell Univ. Press, Ithaca, N.Y

Ohnmeiss, T.E. and I.T. Baldwin. 2000. Optimal defense theory predicts the ontogeny of an induced nicotine defense. Ecology 81:1765-1783.

Ow, M.C., M. Gantar, and J. Elhai. 1999. Reconstitution of a cycad-cyanobacterial association. Symbiosis 27:125-134.

Palm and Cycad Societies of Australia. 1993. The biology, structure, and systematics of the Cycadales. Proc. 2nd Intl. Conf. Cycad Biology, Townsville, Queensland, Australia, 22-28 July 1990.

Peters, R.H., S. Cloutier, D. Dube, A. Evens, P. Hastings, H. Kaiser, D. Kohn, and B. SarwerFoner. 1988. The allometry of the weight of fruit on trees and shrubs in Barbados. Oecologia 74:612-616.

Rillig, M.C., S.F. Wright, M.S. Torn, and K.A. Nichols. 2001. Unusually large contribution of arbuscular mycorrhizal fungi to organic matter pools in tropical forest soils. Plant Soil 233:167-177.

Rogers, R.F. 1995. Destiny's Landfall-A -A history of Guam. Univ. Hawaii Press, Honolulu.

Santa Regina, I. and T. Tarazona. 2001. Nutrient cycling in a natural beech forest and adjacent planted pine in northern Spain. Forestry 74:11-28.

Schenk, H.J., C. Holzapfel, J.G. Hamilton, and B.E. Mahall. 2003. Spatial ecology of a small desert shrub on adjacent geological substrates. J. Ecol. 91:383-395.

Shaw, C.A. and J.M.B. Wilson. 2003. Analysis of neurological disease in four dimensions: Insight from ALS-PDC epidemiology and animal models. Neurosci. Biobehavioral Rev. 27:493-505.

Stone, B.C. 1970. The flora of Guam. Micronesica 6:1-659.

Tsimilli-Michael, M., P. Eggenberg, B. Biro, K. Koves-Pechy, I. Voros, and R.J. Strasser. 2000. Synergistic and antagonistic effects of arbuscular mycorrhizal fungi and Azospirillum and Rhizobium nitrogen-fixers on the photosynthetic activity of alfalfa, probed by the polyphasic chlorophyll a fluorescence transient O-J-I-P. Appl. Soil Ecol. 15:169-182.

van Dam, N.M. and I.T. Baldwin. 2001. Competition 
mediates cost of jasmonate-induced defenses, $\mathrm{N}$ acquisition and transgenerational plasticity in Nicotiana attenuata. Funct. Ecol. 15:406-415.

van der Pij1, L. 2002. Principles of dispersal in higher plants, p. 309-321. In: R.L. Chazdon and T.C. Whitmore (eds.). Foundations of tropical forest biology. Univ. Chicago Press, Chicago.

Viti, R., S. Bartolini, and R. Guerriero. 2003. The influence of sampling from different canopy positions on the evaluation of flower bud anomalies and dormancy. Fruits 58:117-126.

Watanabe, M. 1996. Developmental regulation of ionotropic glutamate receptor gene expression and functional correlations, p. 73-90. In: C.A. Shaw (ed.). Receptor dynamics in neural development. CRC Press, Boca Raton, Fla.

Weiss, J.H., J.Y. Koh, and D.W. Choi. 1989. Neurotoxicity of beta-N-methylamino-L-alanine (BMAA) and beta-N-oxalylamino-L-alanine
(BOAA) on cultured cortical neurons. Brain Res. 497:64-71.

Whiting, M.G. 1963. Toxicity of cycads. Econ. Bot. 17:271-302.

Wiles, G.J. 1992. Recent trends in the fruit bat trade on Guam, p. 53-60. In: D.E. Wilson and G.L. Graham (eds.). Pacific island flying foxes: Proceedings of an international conservation conference. U.S. Fish Wildlife Serv. Biol. Rpt. 90(23).

Willet, W.C. 2002. Balancing life-style and genomics research for disease prevention. Science 296:695-698.

Wilson, J.M., I. Khabazian, M.C. Wong, A. Seyedalikhani, J.S. Bains, B.A. Pasqualotto, D.E. Williams, R.J. Andersen, R.J. Simpson, R. Smith, U.K. Craig, L.T. Kurland, and C.A. Shaw. 2002. Behavioral and neurological correlates of ALS-parkinsonism dementia complex in adult mice fed washed cycad flour. Neuromol. Med. 1:207-221.

Yagi, F. 2004. Azoxyglycoside content and $\beta$-glycosidase activities in leaves of various cycads. Phytochemistry 65:3243-3247.

Young, F.J. 1988. Soil survey of Territory of Guam. U.S. Dept. Agr. Soil Cons. Serv.

Zaninovic, V. 2004. Possible etiologies for tropical spastic paraparesis and human T lymphotropic virus I-associated myelopathy. Braz. J. Med. Biol. Res. 37:1-12.

Zhang, Z.X., D.W. Anderson, N. Mantel, and G.C. Roman. 1996. Motor neuron disease on Guam: geographical and familial occurrence, 1956-85. Acta Neurol. Scand. 94:51-59.

Zotz, G. 2000. Size-related intraspecific variability in physiological traits of vascular epiphytes and its importance for plant physiological ecology. Perspectives Plant Ecol. Evol. Syst. 3:19-28. 